\title{
Adaptive Block Testing
}

\author{
Timo von Oertzen \\ University of the Bundeswehr, Munich and Max Planck Institute for Human \\ Development, Berlin
}

Address correspondence regarding this manuscript to Timo von Oertzen (timo@unibw.de), University of the Bundeswehr, Munich

\begin{abstract}
This article introduces Adaptive Block Testing (ABT), a method to test $N$ units for a binary variable with known baseline probability $p_{i}$ for each unit, assuming that a test is available which may take arbitrary number of units and tests negative if all units are negative, and positive otherwise. A proof is given that the current method is optimal up to rounding. ABT is applicable to screen a large population of patients for the presence of the RNA of a virus, as for example the SARS-CoV-2, using block testing by polymerase chain reactions. ABT uses the block tests and adaptively chooses from the pool participants such that the entropy gain in each test is maximized. For a baseline probability of $1 \%$ of the tested patients to be sick, the method needs 2.4 times less tests than a block testing method with a block size of 10, the optimal block size for a standard block test at a baseline probability of $1 \%$.
\end{abstract}

\section{Keywords: COVID 19, SARS-CoV-2, Block Testing, Adaptive Testing}

\section{Introduction}

We are currently in a pandemic with the Severe Acute Respiratory Syndrom Coronavirus 2 (SARS-CoV-2), a virus that causes the Coronavirus Disease 2019 (COVID-19). There is a general consensus that the virus poses a considerable threat to global health (see for example Hui et al., 2020). As of today, over 2.7 million cases have been reported, with more than 190.000 deaths (Center for System Sciences at the John Hopkins University, 2020). 
A crucial part of fighting the pandemic is testing for the presence of the virus. This is typically done using a reverse transcription polymerase chain reaction (rPT-PCR; Freemann, Walker, \& Vrana, 1999). In a nutshell, the test procedure first multiplies all Ribonucleic acids (RNA) in a sample exponentially. It then compares the retrieved RNA strings with a pregiven pattern, for example, specific markers of the RNA in the SARS-CoV-2. If the pattern is found, the test will give a positive result.

Each PCR takes considerable time and resources. In some situations, when the apriori probability of finding the virus is fairly high and the sample small, there is no way around these costs. An example for such a situation is if the test is used to identify all infected persons who had personal contact to a confirmed case. However, when screening a larger population with a lower probability of infection, as for example the staff in a major hospital or a nursing facility, block tests can be used to reduce the total number of tests. The idea of a block test is that samples from multiple patients can be mixed together, and then a single PCR can be executed on all samples at once. If the result is negative, one knows that none of the patients had the virus; otherwise, one knows that at least one patient is infected (Dorfmann, 1943). This method has also been suggested to be used in the current Corona crisis by Erhard Seifried (in public communications, 2020).

If the a-priori probability that the whole block is healthy is reasonably high, block tests can accelerate testing strongly. If a block size of $K$ is chosen, the expected average number of tests per patient is $\frac{1}{K}$ if all patients are healthy, and $\frac{K+1}{K}$ if at least one patient is sick. If the a-priori probability to be healthy is $p$, the average expected number of tests is therefore $p^{K} \frac{1}{K}+\left(1-p^{K}\right) \frac{K+1}{K}$. The $K$ is usually chosen as a fixed small number (e.g., 5 or 10), but can be adapted to $p$ to reach better performance.

However, the standard strategy to test every single patient if the whole block was tested negatively seems an excess of tests. The current article introduces an adaptive variant of the block test, Adaptive Block Testing (ABT), which allows to reduce the average number of tests by a factor of 7 for a very low probability of $0.05 \%$ to be sick to a factor of 2 for a for a probability of roughly $2 \%$ to be sick, and still reducing the numbers of test to two thirds even for $10 \%$ probability to be sick. This article will show that ABT, up to rounding errors, is optimal in the sense that there exists no other testing strategy that allows a smaller expected number of tests.

The general idea of ABT is to choose samples from the pool such that the expected probability to hit a positive result has maximal entropy (Shannon, 1951), which is reached if the probability is closest to $50 \%$. This idea is analogously to adaptive testing in behavioral sciences (see for example Van der Linden \& Glas, 2010), or active learning in the field of computer science (see Settles, 2010). Since the expected entropy of the tested distribution, in our example the knowledge about the sickness status of the patients in the pool, is reduced exactly by the entropy of each test, and finally must reach zero when the status of all patients is to be learned, this method achieves the minimal possible number of tests. The main difficulty is to choose the tests in a way that (1) the entropy is maximal, and (2) it is possible to keep track of the distribution of sickness in the population to be tested.

Obviously, in block testing it does not suffice for goal (2) to keep track of every person's probability to be sick at every step, since being in one block creates dependencies between the patients. The idea of ABT is to create test sets that are nested in each other as long as the test is positive, i.e., at least one person is sick in all nested sets. This 
allows to eliminate every negatively tested subgroup from all previous groups. If a single person is identified as being sick, we know that he or she has been part of all previous tests and therefore eliminates all information we have learned about these groups. This simplifies our representation of the population's distribution, and allows to keep track of the population's distribution exactly, allowing us to choose every new set according to rule (1). The only source of loss we have in this procedure are rounding errors, since especially in small populations or at the end of the process we have not enough degrees of freedom to reach a test probability of 0.5 exactly. However, ABT minimizes these rounding errors as long as possible by adding small additional sets to each test set.

ABT can not only be used for testing of a virus. For example, when testing microchips which work when stringed in series as long as none of the chips is defect, the same method can be applied. In cognitive psychology, sometimes multiple abilities can be tested in a single task which will only be successful if the participant has all abilities (for example, knows the definition of all terms used in a task). If these can be combined flexibly, then ABT can be used to learn about the participants ability for each subtask with minimal tests. Some group tasks can only be performed if all members of the group have a certain ability. By recombining tested groups, ABT can be used in such situations to quickly identify participants that lack the ability if the tests themselves are equally resource intensive.

In the following, this article will first introduce ABT mathematically, and then provide a pseudo-code version of the core algorithm. Then a simulation study will be presented to validate that $\mathrm{ABT}$ is close to the maximum, and compare it to classical block testing with a fixed block size or even to block testing when using block sizes optimally chosen for the a-priori probability. Some extensions of the algorithm to related problem settings are introduced, and the article closes by a short discussion.

\section{Mathematical Description}

\section{Terminology and Lower Bound for the Tests Needed}

Assume $X=X_{1}, \ldots, X_{N}$ are $N$ dichotomous unknown independent Bernoulli random variables over $\{0,1\}$ with known a-priori probability $P\left(X_{i}=1\right)=p_{i}$. A test $T\left(X_{1}, \ldots, X_{K}\right)$ is available with outcomes in $\{0,1\}$ which is 1 iff all $X_{i}=1$ (where the outcome 1 means a negative test in the example of a sickness). For a set $S \in\left\{X_{1}, \ldots, X_{N}\right\}$, we will use $S$ also for the event that all $X_{i} \in S$ are 1 , and $\bar{S}$ for the complement that at least one element is 0 .

The Entropy of the random variable $X$ is given by $H(X)=$ $\sum_{i=1}^{N}\left(p_{i} \log \left(p_{i}\right)+\left(1-p_{i}\right) \log \left(1-p_{i}\right)\right)$. Obviously, each test result has at most an entropy of $\log (2)$. Since the entropy of the random variable can thus reduce at most by

$\log (2)$ for each step in expectation, $\frac{H(X)}{\log (2)}$ is a lower bound for the expected number of tests needed to learn the outcome of every $X_{i}$. This bound can be reached up to rounding if each test has probability as close to 0.5 as possible, and track is kept of the posterior distribution over $\{0,1\}^{N}$ of all units. The latter is difficult to obtain when choosing arbitrary tests. This article suggest a Las Vegas (Babis, 1979) algorithm that chooses tests in a way that their probability is close to 0.5 and allows to maintain the distribution in a sparse way. 


\section{General Form of the Algorithm}

In its basic form, the algorithm proceeds in the following steps. First, a set $S_{1}$ of $K$ units are chosen from the pool of $N$ units such that $P\left(S_{1}\right)=\prod_{x_{i} \in S_{1}} p_{i}$ is close to 0.5. $S_{1}$ is then tested; if the test returns 1 , all units are marked as having a value of 1 , and the algorithm restarts with the remaining $N-K$ units. Otherwise, a subset $S_{2}$ of $S_{1}$ is chosen such a test on $S_{2}$ condition on the previous result has probability $P\left(S_{2} \mid \overline{S_{1}}\right)$ close to 0.5 . If the test on $S_{2}$ is 1 , all elements of $S_{2}$ are marked as 1 and removed from the set. We know that $S_{1}-S_{2}$ would be tested as 0 now, since the test was zero on $S_{1}$ in total, but all units in $S_{2}$ are one. $S_{1}$ is now set to $S_{1}-S_{2}$, and the algorithm proceeds as if this set has been chose initially, with providing a 0 test result.

If otherwise $\overline{S_{2}}$, a new smaller subset $S_{3} \subset S_{2}$ is chosen as before, again aiming at putting the a-posteriori probability for a test on $S_{3}, P\left(S_{3} \mid \overline{S_{1}}, \overline{S_{2}}\right)$, close to 0.5. The algorithm proceeds analogously from here, always going one step back on a test result of 1 , removing the tested subgroup from all previous sets, and moving one step forward for a test result of 0 . If a set containing exactly one unit is tested to be zero, this unit is marked as 0 , and all other units in the current initial set $S_{1}$ are returned to the general pool. This can be done since all tests results so far are fully explained by the one unit tested to be zero, since it has been an element in all tested sets; so all other elements again are independent and have their respective a-priori probabilities.

The algorithm described so far works, but rounding errors increase as the subsets become small. To reduce these rounding errors, whenever a subset $S_{i+1} \subset S_{i}$ is created, its probability condition on the previous tests (here abbreviated as $T$ ) is chosen as $P\left(S_{i+1} \mid T\right)>$ 0.5 with minimal possible distance to 0.5 . Then, additional units from the pool which have not been used so far are collected in an appendix set $A_{i+1}$. Since these elements have not been in tests with 0 result before, their probability is still the a-priori probability, the highest these elements may have in the process. So they are most suited to fine-adjust the probability of a block test of $T_{i+1}=S_{i+1} \cup A_{i+1}$ to 0.5 . Since the elements of the appendix $A_{i+1}$ are independent, this probability is $P\left(A_{i+1}\right) \cdot P\left(S_{i+1} \mid T\right)$. Note that the next subset is still chosen just from $S_{i+1}$ without the elements in the appendices.

At the end of the process, when no elements are left in the pool, the appendices are left empty, and the initial subset $S_{1}$ is chosen as the whole pool when elements are returned to it. The rounding errors occuring then only occur in this last phase, and their relativ influence goes to zero for large $N$.

\section{Computing the Conditional Probability of the Next Split}

It remains to show how to compute the posterior probability $P\left(S_{i+1} \mid T\right)$ used to choose the for a subset of units in the current smallest test. This is done by the following lemma and theorem:

Lemma 1. Let $X=X_{1}, \ldots, X_{N}$ be a set of dichotomous variables and $S_{1}, \ldots, S_{M} \subseteq X$ such that $S_{i+1} \subset S_{i}$. Let $A_{1}, \ldots, A_{M-1} \subseteq X$ pairwise disjunct sets also disjunct from $S_{1}$, and $T_{i}=S_{i} \cup A_{i}$. Let $S^{\prime}=S_{M-1}-S_{M}$ be the complement of $S_{M}$ with respect to $S_{M-1}$. Then,

$$
P\left(\overline{T_{M-1}}, \ldots, \overline{T_{1}} \mid S_{M}\right)=P\left(\overline{S^{\prime}}\right)+P\left(S^{\prime}\right) \cdot P\left(A_{M-1}\right) \cdot P\left(\overline{T_{M-2}}, \ldots, \overline{T_{1}} \mid S_{M-1}\right)
$$


Proof. Dependend on whether $S^{\prime}$ is true or not, we can decompose the probability using the Theorem of Total Probabilities into

$$
P\left(\overline{T_{M-1}}, \ldots, \overline{T_{1}} \mid S_{M}\right)=P\left(\overline{T_{M-1}}, \ldots, \overline{T_{1}} \mid S_{M}, S^{\prime}\right) P\left(S^{\prime}\right)+P\left(\overline{T_{M-1}}, \ldots, \overline{T_{1}} \mid S_{M}, \overline{S^{\prime}}\right) P\left(\overline{S^{\prime}}\right)
$$

Obviously, if $S^{\prime}$ contains an unit of value zero, then all previous tests were false, so

$$
P\left(\overline{T_{M-1}}, \ldots, \overline{T_{1}} \mid S_{M}, \overline{S^{\prime}}\right)=1
$$

Otherwise, if $S_{M}$ and $S^{\prime}$ are true, then $S_{M-1}$ is true, and $T_{M-1}$ can only be false if $A_{M-1}$ is false, so

$$
\begin{aligned}
P\left(\overline{T_{M-1}}, \ldots, \overline{T_{1}} \mid S_{M}, S^{\prime}\right) & =P\left(\overline{T_{M-1}}, \ldots, \overline{T_{1}} \mid S_{M-1}\right) \\
& =P\left(\overline{T_{M-2}}, \ldots, \overline{T_{1}} \mid S_{M-1}\right) P\left(\overline{A_{M-1}}\right)
\end{aligned}
$$

which can be combined to the statement. Note that if $M \leq 2$, the event is about the empty set to contain no element of zero, which make the statement trivially correct.

With this Lemma, we can compute the probability for any subset $S_{M}$ of $S_{M-1}$ under the condition that the previous tests have all returned a zero result:

Theorem 2. Under the same assumptions as in Lemma 1, the probability for $S_{M}$ to contain only units with value 1 is conditionally on the previous tests $T_{M-1}, \ldots, T_{1}$

$$
P\left(S_{M} \mid \overline{T_{M-1}}, \ldots, \overline{T_{1}}\right)=\frac{P\left(\overline{T_{M-1}}, \ldots, \overline{T_{1}} \mid S_{M}\right) P\left(S_{M}\right)}{P\left(\overline{T_{M-1}}, \ldots, \overline{T_{1}} \mid S_{M}\right) P\left(S_{M}\right)+P\left(\overline{S_{M}}\right)}
$$

Proof. The theorem is a consequence of Bayes' Theorem including the simple observation that

$$
P\left(\overline{T_{M-1}}, \ldots, \overline{T_{1}} \mid \overline{S_{M}}\right)=1
$$

which is true since $S_{M}$ is a subset of all $S_{i}$, and hence of all $T_{i}$, for $i<M$.

Using a recursive procedure to compute $P\left(\overline{T_{M-1}}, \ldots, \overline{T_{1}} \mid S_{M}\right)$ using Lemma 1 allows to compute the posterior probability for each possible set $S_{M}$. For computational purposes, note the following corollary:

Corollary 3. Under the same assumption as in Lemma 1 and writing $O R\left(S_{M}\right):=\frac{P\left(S_{M}\right)}{P\left(\overline{S_{M}}\right)}$ for the odd ratio, we get

$$
O R\left(S_{M} \mid T\right)=O R\left(S_{M}\right) \cdot\left(P\left(\overline{S^{\prime}}\right)+P\left(S^{\prime}\right) z\right)
$$

with

$$
z=P\left(A_{M-1}\right) \cdot P\left(\overline{T_{M-2}}, \ldots, \overline{T_{1}} \mid S_{M-1}\right)
$$

where $z$ does not depend on the choice of $S_{M}$.

The probability of $S_{M}$ is close to 0.5 if the odd ratio is close to 1 , so this representation allows to precompute $z$ once and then choose the best $S_{M}$ easily. 


\section{The Specific ABT Algorithm}

A full version of the algorithm in Java can be found in the Appendix. Here is a short notation in pseudocode:

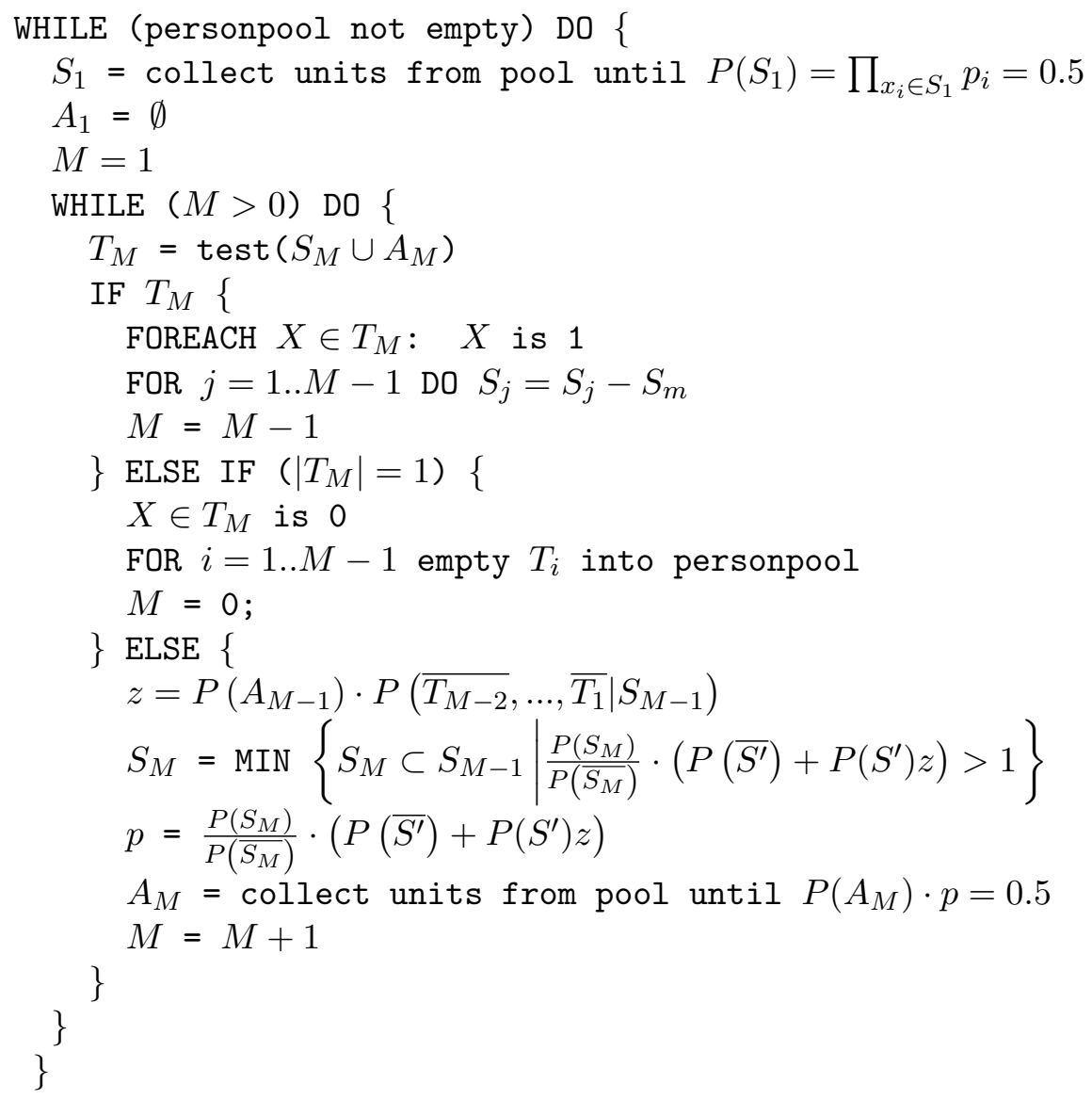

\section{Comparison to Other Methods}

In this section, $\mathrm{ABT}$ will be compared to other methods to evaluate for a number of units whether they satisfy a certain condition, e.g., have the SARS-CoV-2 virus in terms of the expected average number of tests $a$. The naiv testing method is to test each person individually, using exactly one test per unit. As mentioned earlier, the theoretical optimal expected reduction of the entropy in each step is $\log (2)$, which means that the theoretical optimal tests per unit are

$$
a_{o p t}=\frac{H(X)}{N \log (2)}=\frac{-\sum_{i=1}^{N}\left(p_{i} \log \left(p_{i}\right)+\left(1-p_{i}\right) \log \left(1-p_{i}\right)\right)}{N \log (2)}
$$

In our example, assume the a-priori probability for each participant to be healthy is $p=0.99$, i.e., there is a $1 \%$ chance for every participant to have the SARS-CoV-2 virus. Then, the optimal number is $a_{\text {opt }}(0.99)=-\frac{0.01 \log (0.01)-0.99 \log (0.99)}{\log (2)}=0.081$ tests to be taken for each participant. 


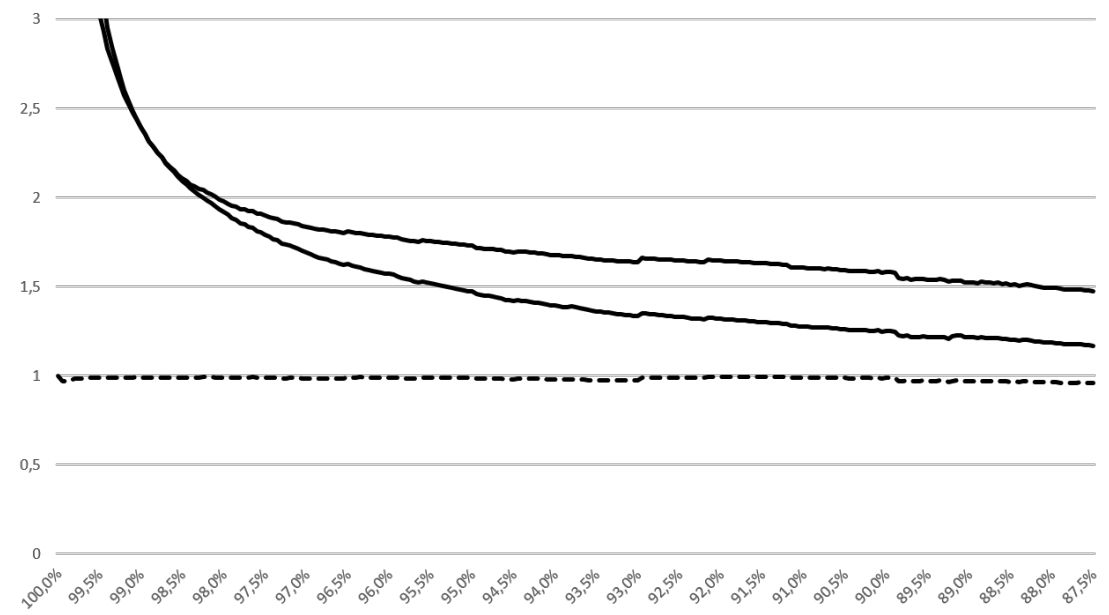

Figure 1. The relative gain of the ABT method compared to a block testing with fixed block size of 10 (upper curve) and optimally chosen block size (lower curve). The dashed line is the relative loss compared to the theoretical optimum. For example, if roughly $1 \%$ of the population have the SARS-CoV-2 virus $(p=0.99)$, using ABT uses less than half of the tests (a factor of 2.35 less) to learn the status of every patient than a classical block test. This is more than 12 times less tests compared to testing every single person of the population.

In a block testing procedure with a fixed block size of $K$, one test is needed if all participants in the block are healthy, and $K+1$ tests are needed if there is at least one sick participant. The average number of tests needed, assuming a fixed a-priori probability $p$, is

$$
a_{K-b l o c k}=p^{K} \frac{1}{K}+\left(1-p^{K}\right) \frac{K+1}{K}=\frac{K+1}{K}-p^{K}
$$

In our example above, with $K=10$ and $p=0.99$, the average number of tests needed with this design is $a_{10-b l o c k}(0.99)=0.196$ tests.

If a reasonable guess for $p$ is available, then the above equation of $a_{K-b l o c k}$ can be minimized for $K$. For simplification, and since $K$ is rounded anyway, an approximation of the value can be chosen which ignores the one additional test needed in the case that a wrong test has been found. Then, the optimal $K$ is

$$
K_{o p t}=\frac{1}{2}+\sqrt{\frac{1}{4}-\frac{1}{\ln (p)}}
$$

In our example with $p=0.99$, this value evaluates to 10.49 , so the performance in this example is again 0.196 .

To compare these approaches to ABT, considering the rounding error, a simulation has been performed of the ABT for $p$ ranging from $100 \%$ to $87.5 \%$ in steps of $.05 \%$, with 100,000 repetitions for each situation. Figure 1 shows the relative gain of ABT compared to a block testing with fixed block size of 10 (upper curve) and optimally chose block size (lower curve). The dashed line is the relative loss compared to the optimum.

As can be seen, ABT is consistently better than classical block tests. In our previous example, when one assumes that $1 \%$ of a population has the SARS-CoV-2 virus, ABT 
would use a factor of 2.35 less tests (which is, $42.5 \%$ of the tests) that would be used for a classical block test. One can see that most of the time, ABT uses roughly as many tests as the theoretical optimum. There is a non-monotonicity in the curve roughly at $89.5 \%$, where ABT drops from $1.7 \%$ points. This is because there is no good set size for the first set $S_{1}$ in the algorithm; all possibilities at this a-priori probability introduce a small rounding error.

At roughly $88.5 \%$, the theoretically optimal number of tests, and with it the tests needed with the ABT method, is half the number used when testing every single patient, and at $50 \%$, testing every single patient is the best option.

\section{Extensions of ABT to Similar Problem Settings}

In this section, some plausible small alterations of the problem setting are introduced in which further restriction apply or assumptions are less certain. For each of these variants of the problem, it will be discuss how the algorithm can be adapted to cope with the situation.

\section{Individual a-priori Probabilities}

In the simulation example, the situation was discussed in which the probability for a test result of one was the same for all units of observation. However, the algorithm is framed more generally, allowing to assign a different a-priori probability to each unit.

To minimize the rounding error in the tests, the units are sampled into the appendices in descending order of probability, allowing for most precise approximation of the 0.5 probability for the major part of the population, and using the units that allow least fine-tuning in the end of the algorithm, where rounding errors increase anyway because of the lack of more units to put in the appendices. In theory, one may use a subset sum algorithm in each step to find the optimal subsets and appendices (Soma \& Toth, 2002). However, since the problem is NP hard in general, this would increase the running time of the algorithm considerably without major benefits, considering how close to the optimal number of samples the algorithm is anyway. However, for very large populations and if the test is considerably more resource intensive than running the algorithm, this alteration could be an option.

\section{Variable a-priori Probabilities}

The algorithm in the form presented above assumes known a-priori probabilities, where a deviation from this will not change the correct outcome, but may result in a sub-optimal choice of the tested blocks. The same problem occurs when using a classical block testing algorithm with optimal block size, because the block size selected will only be optimal with respect to the assumed a-priori probability; any fixed block size chosen by rule of thumbs are obviously even worse.

However, the algorithm can be changed to adapt to new knowledge about the probability of the test as the testing moves along if the participants are from the same distribution. Assume that all units come from the same distribution, i.e., are independent Bernoulli draws with the same probability. One can use a Beta distribution as a prior for the probability of each single unit. To approximate the posterior distribution of units that have not been tested before, whenever a unit is marked as '1' or '0', we update the Beta distribution with 
this new unit. As the algorithm proceeds, the empirical probability of the tested population will overrule the prior, and the probability will converge to the true population value.

This process can be extended to a situation where different subpopulations exist that have unknown, but likely different posterior probabilities. In this situation, the algorithm only updates the Beta distribution of the units that are in the same subpopulation as the unit which just has been marked as '1' or ' 0 '.

Note that the version of the algorithm introduced in the previous sections with a fixed probability for each unit is a special case of this extension, by setting the strength of the prior Beta distribution for each unit's probability to infinity. On the other end of the scale, we may use a Beta 1,1 distribution (which is flat in the area from zero to 1 ) to create a situation where we have no a-priori idea at all about the probabilities of each unit's test. In this case, since the expected probability is 0.5 , the algorithm starts by testing individual units until the a-posteriori becomes known better.

\section{Maximal Number of Tests per Unit}

In practice the algorithm will typically assign each unit only to a limited number of tests in total, with an average of at most two test assignments for each unit. For example, with an a-priori probability of $p=99.95 \%$, the average number of tests that each unit was assigned to was 1.677 in the simulation; with a probability of $p=98 \%$, the average number of tests was 1.985 . However, in theory this amount can be large for some exceptional cases, which may not be possible to do in practice. For instance, in our virus example each sample taken from a person may not be large enough to allow arbitrary number of tests.

To work with a maximal number of tests per unit, one can count the tests taken and, as soon as only one test is left for a unit, test this unit separately regardless of the expected probability. This unit will be marked according to the test result and removed from the procedure. If the unit test returns 1 for an element of one of the regular sets, we put every other unit currently in the sets and appendices back in the pool, since all previous negative tests are accounted for by this unit. If the unit is in the appendix of the current test, the other units in the appendix are returned to the pool, and a new appendix is chosen (which may again recruit from the returned units). If the unit test returned 0 , we just remove the unit from its current set or appendix and continue with the remaining state of the algorithm; note that the a-posteriori probabilities that are affected by the change will only be computed later, since the one-before-last test can only have occurred on $A_{M}$ or $S_{M}$.

Note that it is possible that multiple elements reach the maximal number of tests at the same time; then, a single ' 1 ' test either in an appendix or regular set is sufficient to the consequences as described above, with the reaction to the regular set of course taking precedence if both sets contain a '1' unit.

\section{Parallel Testing}

It is often possible to conduct parallel testing, for example if multiple labs participate in the testing of a whole population. In this case, the optimal number of tests can be achieved by distributing the test units over all available labs, and use the testing procedure described in this article in each lab separately. Note that the relative gain does not change in this situation. However, if the number of parallel testing facilities are anyway as large as the number of tests do be done, so that each testing facility only receives zero to two test 
units, there is no need for block testing with any method. Note, however, that considering that tests are expensive, it may be worth to delay testing in each testing facility until some test units (depending on the probability, roughly 10 to 30 tests) have accumulated, and then test all of these with the block testing procedure to save testing resources.

\section{Discussion}

The current article introduced a test procedure to use block tests adaptively, where each block test reveals whether at least one element of a set has a certain property. The method can for example be used to test a large population for presence of the Cov-SARS-2, reducing the number of required test to less than one half if the probability of being sick is close to $2 \%$ for example, much more for lower probabilities and less for higher probabilities.

The method is based on maximizing the entropy gain in each test by taking the probability of the test as close as possible to 0.5 while maintaining a correct representation of the distribution for all units in the population. This is done by testing nested subsamples on negative tests.

A proof was presented that the method is optimal up to rounding errors. It was also demonstrate in a simulation that the method deviates less than $3 \%$ from the optimum because of these rounding errors.

The method can easily be extended to cases where each unit comes with its own probability of failing, where the probability of failing is not (or at least not certainly) known initially, or where there exist a maximal number of tests.

There are two limitations to consider. Firstly, when enforcing a maximal number of tests that each unit can be assigned to, the test procedure will loose optimality. Even though most units are tested infrequently (with an average of two assignments), it is possible that the number of tests could be improved by using a different adaptation of the algorithm as was done in this article. Secondly, the small loss of less than $3 \%$ of additional test due to rounding may potentially be improved, for example by using a full subset sum algorithm at each selection step. However, this minimal gain will only be worthwhile if taking tests is considerably more expensive, and probably only when planning to test very large populations.

An implementation of the testing procedure can be found at https://osf.io/5vksh/. This program stops at every tests and asks the user to input the test result, so that it can directly be used to test Corona cases. The source code for the algorithm is given in the Appendix.

\section{References}

Babis, L. (1979). Monte-carlo algorithms in graph isomorphism testing (Tech. Rep. No. 79-10). Montreal, Quebec H3T 1J4, Canada: University de Montreal.

Center for System Sciences at the John Hopkins University. (2020). Coronavirus covid-19 global cases. retrieved from https://coronavirus.jhu.edu/map.html.

Dorfmann, R. (1943). The detection of defective members of large populations. The Annals of Mathematical Statistics, 14, pp. 436-440.

Freemann, W., Walker, S., \& Vrana, K. (1999). Quantitative rt-pcr: pitfalls and potential. BioTechniques, 26(1), 112-122. 
Hui, D., Azhar, E., Madan, T., Ntoumi, F., Kock, R., \& DAr, O. e. a. (2020). The continuing 2019ncov epidemic threat of novel coronaviruses to global health - the latest 2019 novel coronavirus outbreak in wuhan, china. International Journal of Infectious Diseases, 264-266.

Settles, B. (2010). Active learning literatures survey (Computer Science Technical Report No. 10). Wisconsin: University of Wisonsin.

Shannon, C. (1951). Prediction and entropy of printed english. Bell Systems Technical Journal, $30(1), 50-64$.

Soma, N. Y., \& Toth, P. (2002). An exact algorithm for the subset sum problem. European Journal of Operational Research, 136, 57-66.

Van der Linden, W., \& Glas, C. (2010). Elements of adaptive testing (No. 3). New York: Springer. 


\section{Appendix 1}

This appendix gives the full java code to do the method. It does include individual a-priori probabilities, but it does include the other extensions discussed in the Extensions Section. Please contact the author if you need help implementing any of these extensions.

The program only uses the standard java utility package java.util. The code consists of three classes which by convention should be put in different files. The first two classes are structure classes only, the main algorithm is in the 'testing' method of the 'World' class.

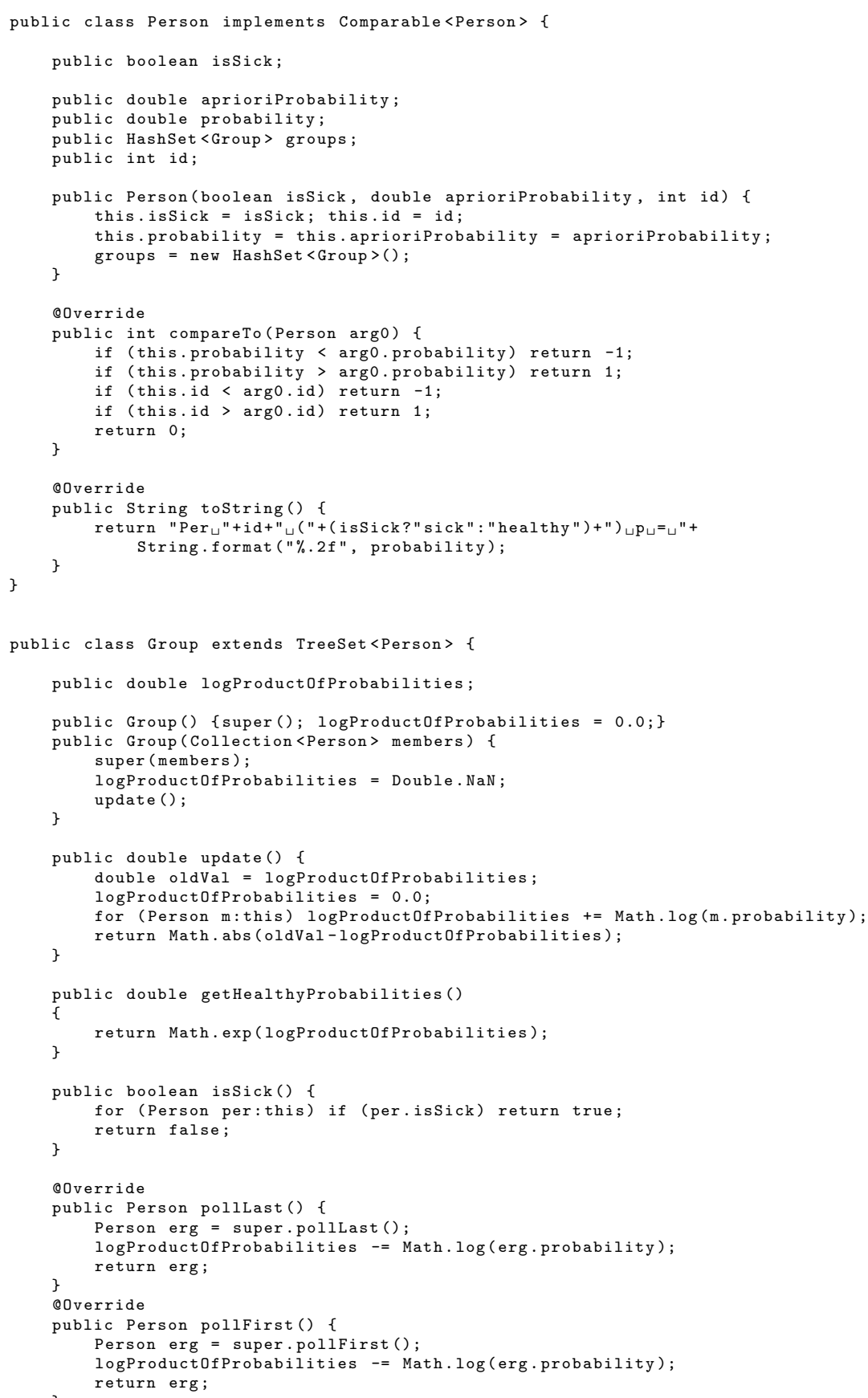




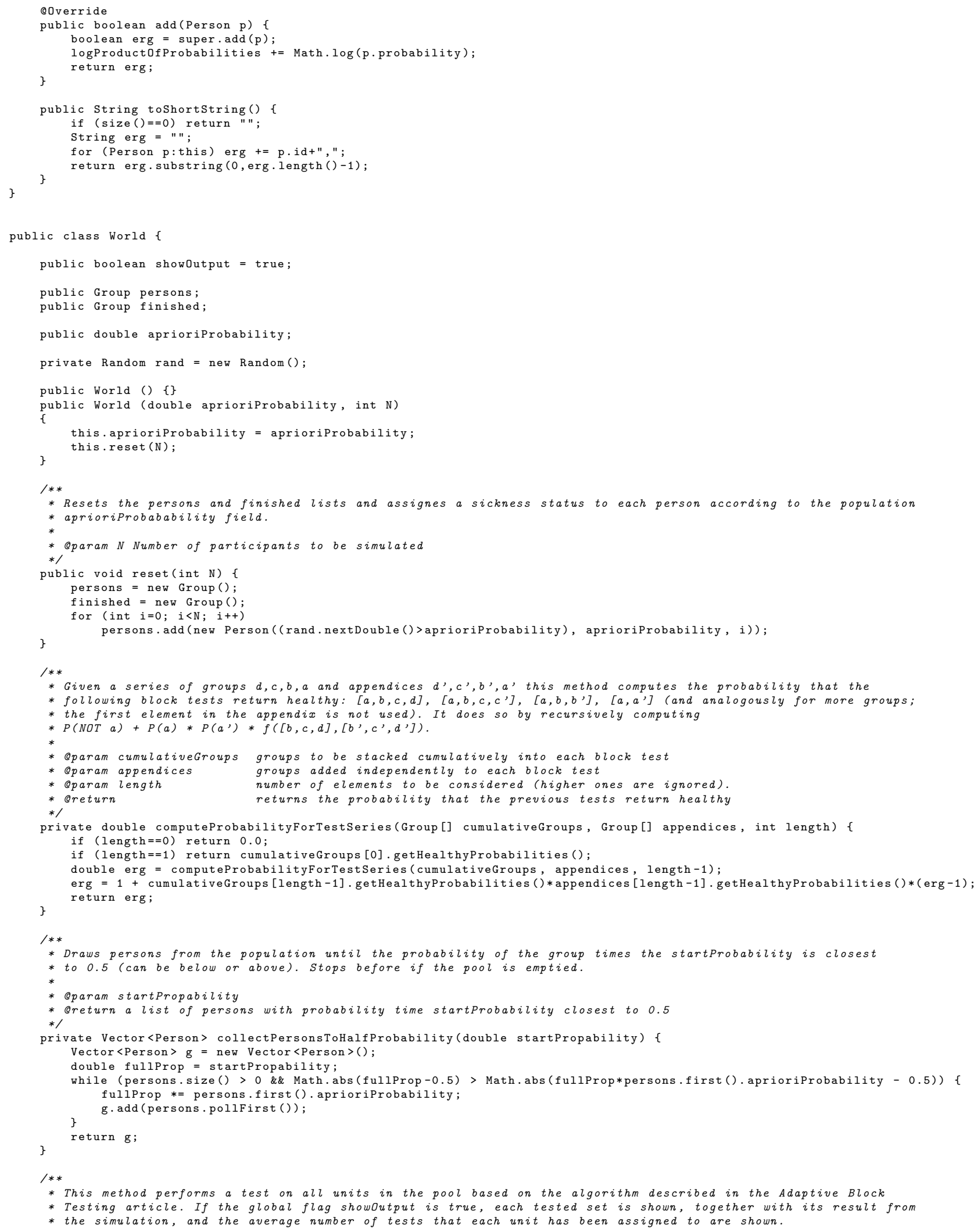




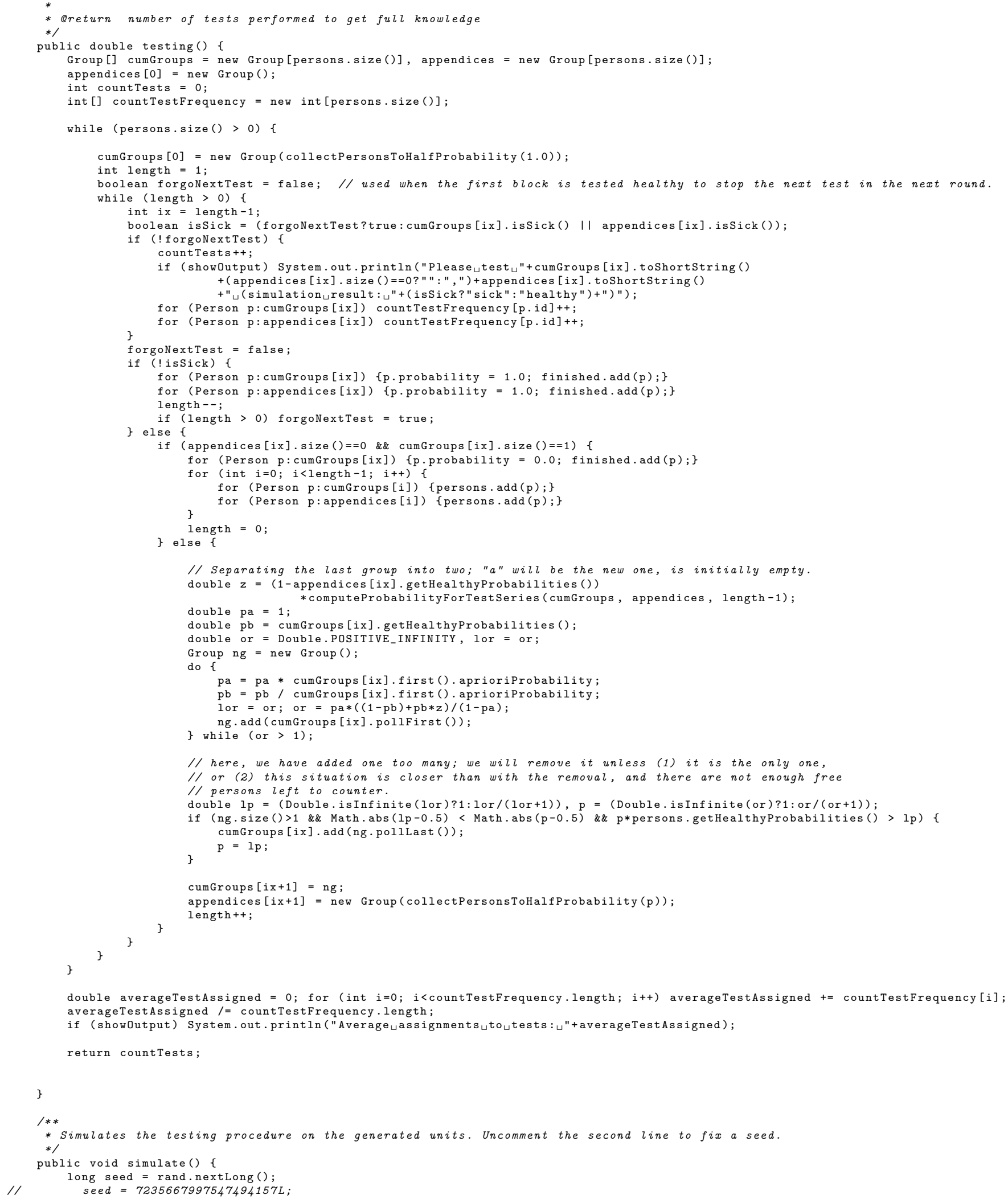




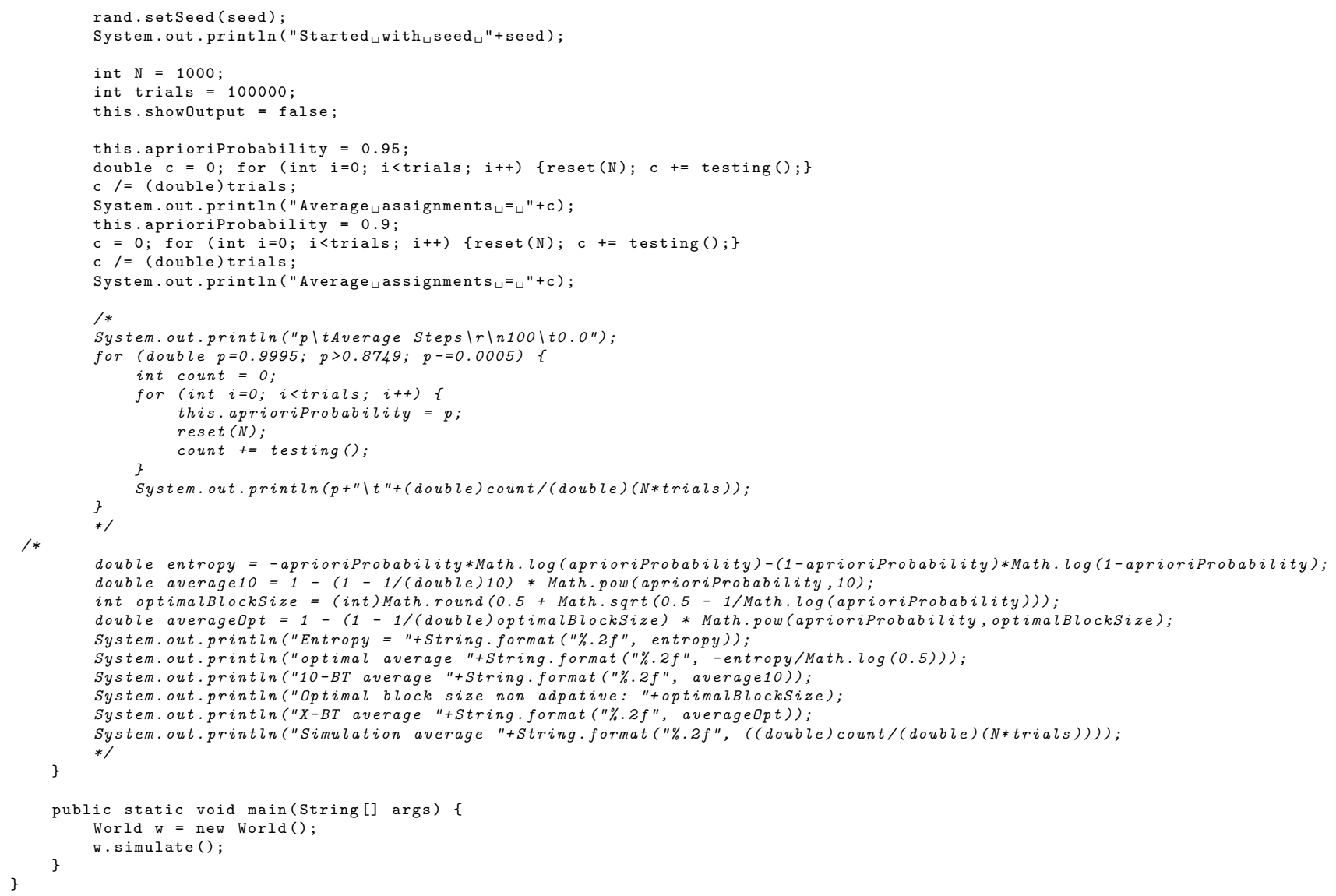

